Référence de la contribution : Chrstian Grappe, « Hébreux et la tradition paulinienne », in : Jens Schröter, Simon Butticaz, Andreas Dettwiler (dir.), Paulusrezeptionen im frühen Christentum - Receptions of Paul in Early Christianity (Beihefte zur Zeitschrift für die neutestamentliche Wissenschaft 240), Berlin, Walter de Gruyter, 2018, p. 461-483.

Résumé français : la question des rapports entre Hébreux et la tradition paulinienne est complexe. Elle est étudiée ici à partir de quatre angles d'approches différents. C'est l'hypothèse d'un rattachement secondaire de l'écrit à cette tradition au cours du second siècle et par le biais d'un billet additionnel (He 13,19.22-25) qui est finalement retenue.

\title{
[461] Hébreux et la tradition paulinienne
}

\author{
Christian Grappe (Strasbourg)
}

English Abstract: The question of the relationships between Hebrews and the Pauline tradition is a complex one. It is studied here from four different angles of approach. It is the hypothesis of a secondary incorporation in that tradition by means of the addition of a short bill (He 13,19.22-25) that is finally retained.

Mots clefs : postscriptum ; $P^{46}$; canon ; Paul ; tradition paulinienne ; épître aux Hébreux.

Avant même d'aborder le sujet que nous traiterons, nous voudrions proposer une remarque préliminaire dont la suite du propos montrera, nous l'espérons du moins, la pertinence dans le cadre d'une réflexion portant sur les réceptions de Paul au sein du christianisme naissant.

Cette remarque est la suivante : le sujet en lui-même est le reflet d'une évolution dans le recherche car, très longtemps, la question qui s'est posée était beaucoup plus directe et se serait formulée ainsi «Hébreux et Paul». L'enjeu était de savoir si Paul était l'auteur de l'écrit ou non. La question de l'apostolicité de l'écrit - et, en rapport avec elle, celle de son lien avec Paul - était au centre du débat. Les acquis de la recherche historico-critique ont permis d'établir de manière définitive, il faut l'espérer, que Paul ne saurait être tenu pour l'auteur d'Hébreux. Dès lors, le débat s'est déplacé et la question ici posée reflète cet état des choses. En s'interrogeant sur «Hébreux et la tradition paulinienne », on prend acte de la distance existant entre l'écrit et l'apôtre et, dès lors, on étudie la relation d'Hébreux avec la tradition paulinienne comme on pourrait aussi scruter sa relation avec d'autres courants du mouvement chrétien naissant, exercice auquel se livrait déjà Ceslas Spicq, avec beaucoup d'acribie, dans son commentaire paru en $1952 .{ }^{1}$ Tout cela illustre le fait que le débat s'est bel et bien déplacé. Hébreux est étudié en fonction de son message propre, bien davantage qu'en fonction de son lien avec Paul, qui est devenu aujourd'hui une question collatérale, voire relativement marginale. Il n'en demeure pas moins que la question n'est aucunement dénuée de pertinence et est même justifiée par la finale de l'écrit lui-même, nous aurons l'occasion d'y revenir.[462]

[461] $]^{1}$ Ceslas Spicq, L'Epître aux Hébreux. I. Introduction, EtB 23 (Paris : Gabalda, 1952), 92-166. Il étudie notamment et successivement les rapports d'He avec le quatrième évangile (109-138), 1 P (139-144) et les épîtres pauliniennes (144-168). [462] 
Dès les temps les plus anciens, les rapports d'Hébreux à la tradition paulinienne ont fait l'objet d'hypothèses à la fois nuancées et complexes qui illustrent quelques-unes des difficultés qui surgissent dès lors que l'on cherche à se situer dans le débat relatif à l'évaluation de ces rapports.

L'Histoire ecclésiastique d'Eusèbe de Césarée s'avère ici précieuse à un double titre car elle nous a conservé à la fois le témoignage de Clément d'Alexandrie (circa 150-215) et celui d'Origène (185-253).

Eusèbe indique en effet que, dans les Hypotyposes, Clément d'Alexandrie « dit [...] que l'Epître aux Hébreux est de Paul et qu'elle a été écrite aux Hébreux en langue hébraïque, mais que Luc, après l'avoir traduite avec soin, l'a éditée pour les Grecs ; c'est pourquoi on trouve la même apparence à la traduction de cette épître et aux Actes $» .^{2}$ Clément poursuit en affirmant que, si «elle ne porte pas l'inscription "Paul apôtre" ", c'est que, " en l'adressant aux Hébreux qui avaient une prévention contre lui et qui le soupçonnaient, ce fut d'une manière très prudente qu'il ne les rebuta pas dès le début, en y mettant son nom $»{ }^{3}$ Il ajoute encore que, "puisque le Seigneur, qui était apôtre du Tout-Puissant, fut envoyé aux Hébreux, ce fut par modestie que Paul, comme il avait été envoyé aux Gentils, ne s'intitula pas apôtre des Hébreux, à la fois à cause du respect pour le Seigneur et parce qu'il s'adressait lui aussi aux Hébreux par surcroît, étant le héraut et l'apôtre des Gentils ». ${ }^{4}$

Comme on le voit ici, l'absence du nom de Paul au sein de l'écrit et dans sa suscription interpelle Clément d'Alexandrie qui tient cependant à maintenir son origine paulinienne en faisant intervenir les précautions qu'aurait prises le Tarsiote pour ne pas ruiner d'emblée son propos, que ce soit par absence de prudence ou par immodestie.

Toujours selon le témoignage d'Eusèbe, Origène se montre à la fois plus perspicace et plus prudent, renonçant à une origine directement paulinienne mais posant néanmoins une claire influence paulienne :

« [11] Le caractère du style de l'Epître intitulée Aux Hébreux n'a pas la simplicité dans le discours de l'apôtre [Paul] qui confesse lui-même être simple dans le langage [2 Co 11,6], c'est-à-dire sa phrase, mais l'épître est très grecque par la composition du style, et tout homme capable de juger les différences de style le reconnaîtrait. [12] D'ailleurs, que les idées de l'épître sont admirables et ne sont pas inférieures à celles des lettres apostoliques incontestées, cela aussi, quiconque prête attention à la lecture des lettres des apôtres [463] pourra convenir que c'est vrai. » [13] [...] « Pour moi, si je donnais mon avis, je dirais que les pensées sont de l'apôtre, mais que le style et la composition sont de quelqu'un qui rapporte la doctrine de l'apôtre, et pour ainsi dire d'un rédacteur qui écrit les choses dites par le maître. Si donc quelque Eglise tient cette lettre pour l'œuvre de Paul, qu'elle soit félicitée même pour cela, car ce n'est pas par hasard que les anciens l'ont transmise comme étant de Paul. [14] Mais qui a écrit la lettre ? Dieu sait la vérité. La tradition qui est venue jusqu’à nous parle de certains selon lesquels Clément, qui a été évêque de Rome, a écrit cette lettre, d'autres, d'après lesquels c'est Luc, l'auteur de l'Évangile et des Actes. $\rangle^{5}$

Cette longue citation, présentée comme telle par Eusèbe de Césarée dans son Histoire ecclésiastique, confirme à quel point les questions relatives à l'origine et à l'auteur d'Hébreux sont à la fois anciennes et difficiles.

\footnotetext{
${ }^{2}$ Eusèbe de Césarée, Histoire ecclésiastique 6,14,1 (trad. Gustave Bardy in Eusèbe de Césarée, Histoire ecclésiastique. Livres V-VII. Texte grec, traduction et notes, SC 41 [Paris : Cerf, 1955], 106).

${ }^{3}$ Eusèbe de Césarée, Histoire ecclésiastique 6,14,2 (trad. ibid., 106-107).

${ }^{4}$ Eusèbe de Césarée, Histoire ecclésiastique 6,14,3 (trad. ibid., 107). [463]

${ }^{5}$ Eusèbe de Césarée, Histoire ecclésiastique 6,25,11-14 (trad. ibid., 128).
} 
Dans l'histoire de l'exégèse, de nombreuses propositions ont été faites en vue d'identifier son auteur : Paul - opinion dont fait état Origène en la rapportant, qui plus est, aux « anciens »-; Clément de Rome ; Luc - autres options qu'il présente -, mais encore Barnabas, ${ }^{6}$ Philippe, ${ }^{7}$ Silas, ${ }^{8}$ Apollos, ${ }^{9}$ Aquila ou plus encore Priscille, ${ }^{10}$ sans oublier Pierre, ${ }^{11}$ Aristion, ${ }^{12}$ Jude, ${ }^{13}$ Epaphras, ${ }^{14}$ Timothée, ${ }^{15}$ Marie, ${ }^{16}$ et d'autres encore sans doute. [464]

La prudence d'Origène illustre son indépendance d'esprit et sa rigueur. Elle fait d'ailleurs figure d'exception au sein de la chrétienté orientale car, comme cela est observé régulièrement, l'authenticité paulinienne était largement admise en son sein. C'est ce qu'attestent notamment, outre ceux des «anciens» et de Clément d'Alexandrie, les témoignages de Denys d'Alexandrie ${ }^{17}$ pourtant disciple d'Origène, d'Eusèbe de Césarée,${ }^{18}$ de Jean Chrysostome, ${ }^{19}$ de Théodoret, ${ }^{20}$ de Théodore de Mopsueste, ${ }^{21}$ d'Aphraate, ${ }^{22}$ d'Ephrem, ${ }^{23}$ de Grégoire de Nazianze, ${ }^{24}$ de Basile de Césarée de Cappadoce,${ }^{25}$ de Grégoire de Nysse, ${ }^{26}$ d'Apollinaire de Laodicée ${ }^{27}$ et d'Amphiloque. ${ }^{28}$

En Occident, et cela peut paraître quelque peu paradoxal - nous y reviendrons -, la réception d'Hébreux s'est avérée beaucoup plus timide, de même que son attribution à Paul. Hébreux y est pourtant connue de façon très précoce - et apparemment nettement plus tôt qu'en Orient où elle n'est évoquée qu'au début du III $^{\mathrm{e}}$ siècle avec la mention qu'en fait Clément d'Alexandrie dans ses Hypotyposes - puisque déjà la Première épitre de Clément en fait

\footnotetext{
${ }^{6}$ Tertullien, De pudicitia 20; Franz Dibelius, Der Verfasser des Hebräerbriefes. Eine Untersuchung zur Geschichte des Urchristentums (Strassburg, Heitz, 1910), 43-58; Karl Bornhäuser, Empfänger und Verfasser des Hebräerbriefes, BFChTh 35/3 (Gütersloh : Bertelsmann, 1932), 78-80, et de très nombreux auteurs dont Spicq, Hébreux (note 1), n. 8, 199-200, dresse une liste impressionnante.

${ }^{7}$ Ainsi William M. Ramsay, "The Date of Autorship of the Epistle to the Hebrews », The Expositor Fifth series 9.6 (1899) : 407-422, qui en a convaincu d'autres (voir Spicq, Hébreux (note 1), n. 5, 203-204.

${ }^{8}$ Thomas Hewitt, The Epistle to the Hebrews, TNTC 15 (Grand Rapids, MI : Eerdmans, 1977).

${ }^{9}$ Martin Luther, «Autor epistulae ad Hebreos, quisquis est, sive Paulus, sive ut ego arbitror Apollos, eruditissime allegat hunc locum », WA 44, 709 ; Spicq, Hébreux (note 1), 210-219; Jean Héring, L'épître aux Hébreux, CNT XII (Neuchâtel, Paris: Delachaux \& Niestlé, 1954), 11-12; 126 ; Albert Vanhoye, « Hebräerbrief », TRE 14 (1985), 494-505, ici 496, lignes 38-42 (il reconnaît en même temps que l'hypothèse reste indémontrable).

${ }^{10}$ Adolf von Harnack, « Probabilia über die Adresse und den Verfasser des Hebräerbriefes », ZNW 1 (1900) : 16-41; Ruth Hoppin, Priscilla, Author of the Epistle to the Hebrews, and Other Essays (New York : Exposition, 1969).

${ }^{11}$ Adam Welch, The Autorship of the Epistle to the Hebrews (Edinburg : Oliphant, Anderson and Ferrier, 1898), $1-33$.

12 John Chapman, « Aristion, author of the Epistle to the Hebrews », RBen 22 (1905) : 50-64.

${ }^{13}$ André-Marie Dubarle, « Rédacteur et destinataires de l'épître aux Hébreux », RB 48 (1939) : 506-529.

${ }^{14}$ Charles P. Anderson, « The Epistle to the Hebrews and the Pauline Letter Collection », HTR 59 (1966) : 429438. [464]

${ }^{15}$ John D. Legg, « Our Brother Timothy. A Suggested Solution top the Problem of the Authorship of the Epistle to the Hebrews », EvQ 40 (1968) : 220-223.

${ }^{16}$ Josephine Massyngbearde Ford, «The Mother of Jesus and the Authorship of the Epistle to the Hebrews », BiTod 82 (1976) : 683-694.

${ }^{17}$ Denis d'Alexandrie, Lettre à Fabien, citée par Eusèbe de Césarée, Histoire ecclésiastique 6,41,6.

${ }^{18}$ Eusèbe de Césarée, Histoire ecclésiastique 3,3,5.

${ }^{19}$ Jean Chrysostome, In Epistulam ad Hebrceos, praefatio (PG 63, col. 9-14).

${ }^{20}$ Théodoret de Cyr, Interpretatio Epistoloe ad Hebraeos 542 (PG 82, col. 675A).

${ }^{21}$ Théodore de Mopsueste, In Epistula Pauli ad Hebrceos commentarii fragmenta (PG 66, col. 952A).

${ }^{22}$ Aphraate, Exposés 1,$16 ; 2,14 ; 8,7 ; 13,13$ (dans tous ces passages, «l'Apôtre » désigne manifestement Paul).

${ }^{23}$ Ephrem, In Genesim 21... (voir références complémentaires chez Spicq, Hébreux [note 1], 174, note 2).

${ }^{24}$ Grégoire de Nazianze, Poemata dogmatica I,12,35 (PG 37, col. 474, 1. 10).

${ }^{25}$ Basile de Césarée, Homeliae I in Psalmos XIV, 1 (PG 29, col. 253, 1. 4).

${ }^{26}$ Grégoire de Nysse, Contre Eunome I (PG 45, col. 369D) ; II (PG 45, col. 540D).

${ }^{27}$ Apollinaire de Laodicée, De la foi 38.

${ }^{28}$ Amphiloque, Iambes à Seleucus 300-307 (PG 37, 1596A-1597A).
} 
usage et paraphrase l'écrit à plusieurs reprises. ${ }^{29}$ La parenté la plus frappante est constituée [465] par la reprise, en 1 Clém 36, du thème du grand prêtre secourable (voir surtout He 2,18), d'éléments présents en He 1,3-4 (v. 2) et de trois des citations que l'on trouve en He 1,5-14, celles du Ps 103,4 LXX (1 Clém 36,3 // He 1,7), du Ps 2,7 LXX (1 Clém 36,4 // He 1,5) et du Ps 109,1 LXX (1 Clém 36,5 // He 1,13). Or il est à noter que, malgré sa dépendance littéraire forte à son endroit, Clément ne cite jamais He en tant que tel et ne l'impute pas à Paul.

Vers le milieu du $\mathrm{II}^{\mathrm{e}}$ siècle, Hermas, qui se fait le chantre d'une attitude plus souple que celle des rigoristes en admettant la possibilité d'une unique pénitence post-baptismale, elle-même soigneusement encadrée, refuse toute nouvelle pénitence en cas de récidive, sans doute sous l'influence d'He 6,4-6, qui est en fait plus catégorique et qui a pu, en l'occurrence, imposer à Hermas des limites à sa propre mansuétude. ${ }^{30}$

Le canon de Muratori, si du moins l'on maintient son origine romaine et sa datation haute aux alentours de l'an 190, pourrait être, quant à lui, un témoin de la gêne relative occasionnée à Rome par un écrit dont on ne reconnaissait pas l'origine paulinienne et qui, de plus, proscrivait la possibilité d'une repentance post-baptismale dès lors que l'on n'en proposait pas l'interprétation très souple que l'on rencontre chez Hermas. ${ }^{31}$

Il n'empêche qu'Hippolyte de Rome, qui n'est pas non plus un défenseur de l'authenticité paulinienne, emprunte des thèmes caractéristiques d'He, parmi lesquels celui du « roi et prêtre parfait qui fut le seul à accomplir la volonté de son père ${ }^{32}$ et la mise en tension des sanctuaires céleste et terrestre, le second étant conçu, à la lumière d'Ex 25,40, comme l'ombre du premier. ${ }^{33}$

Cela étant, d'autres auteurs occidentaux ignorent totalement $\mathrm{He}$ ou en font relativement peu de cas, qu'il s'agisse d'Irénée, de Tertullien, qui en parle néanmoins, tient l'écrit pour l'œuvre de Barnabé et indique qu'il est plus généralement reçu que le Pasteur d'Hermas, ${ }^{34}$ de Cyprien de Carthage, de l'Am[466]brosiaster... ${ }^{35}$ Il semble ainsi qu'en Occident He ait été largement délaissée, sans doute au premier chef parce que, contrairement à ce qui se passait dans la chrétienté orientale, l'on doutait de son authenticité apostolique, paulinienne en l'occurrence. ${ }^{36}$ Jérôme apparaît, sur ce point, comme le témoin d'un tournant. Il rappelle dans un premier temps qu'He - depuis Gaïus - est tenu pour un écrit non paulinien par les

\footnotetext{
${ }^{29}$ Ainsi, outre les parallèles entre 1 Clém 36 et He 1,3.4.7.13;2,18; 3,1, 1 Clém 9,3 // He 11,5 (évocation similaire d'Hénoch) ; 1 Clém 10,7 // He 11,17 (exaltation de la foi d'Abraham offrant son fils) ; 1 Clém 12,1// He 11,31 (mise en exergue de la foi de Rahab) ; 1 Clém 17,1 // He 11,37 (évocation de ceux qui ont erré en peaux de chèvres et de brebis) ; 1 Clém 19,1-2 // He 12,1 (leçon similaire tirée du témoignage des pères dans la foi) ; 1 Clém 27,1 // He 10,23 (Dieu fidèle dans ses [465] promesses) ; 1 Clém 27,2 // He 6,18 (impossibilité pour Dieu de mentir) ; 1 Clém 27,4 // He 1,3 (rôle de la parole dans la constitution de l'univers)...

${ }^{30}$ C'est en tout cas ce que pense Spicq, Hébreux (note 1), 178, à la suite de Marie-Joseph Lagrange, Introduction à l'étude du Nouveau Testament. I ère partie. Histoire ancienne du Canon du Nouveau Testament (Paris: Gabalda, 1933), 36-37.

${ }^{31}$ Kurt Aland and Barbara Aland, The Text of the New Testament. An Introduction to the Critical Editions and to the Theory and Practice of Modern Textual Criticism, trad. Eeroll F. Rhodes (Grand Rapids, MI : Eerdmans ; Leiden : Brill, 1987), 49, explique ainsi son absence dudit Canon de Muratori.

${ }^{32}$ Hippolyte de Rome, Commentaire sur Daniel 4,30,9.

${ }^{33}$ Hippolyte de Rome, Commentaire sur Daniel 1,17,2 (d'après le slavon).

${ }^{34}$ Tertullien, De pudicitia 20. [466]

${ }^{35}$ Voir à ce sujet Spicq, Hébreux (note 1), 180-184.

${ }^{36}$ Voir à ce sujet Spicq, Hébreux (note 1), 180-184. Il nous semble que, dans sa monographie, Clare K. Rothschild, Hebrews as Pseudepigraphon. The History and Significance of the Pauline Attribution of Hebrews, WUNT 235 (Tübingen: Mohr Siebeck, 2009), 29-33, majore l'importance du fait qu'He est connu dans la sphère occidentale et minore l'importance du fait que l'écrit n'y est pas pour autant attribué à Paul. Or la distinction a toute son importance dans le débat relatif à l'authenticité paulinienne de l'écrit.
} 
Romains ${ }^{37}$ puis en vient à reconnaître sa canonicité, qu'il fonde non pas sur l'argument d'une authenticité putative mais sur son usage désormais très large au sein des assemblées ecclésiales. ${ }^{38}$ Il rangera finalement, avec Augustin et Innocent $\mathrm{I}^{\mathrm{er}}$ et au nom de l'usage ecclésiastique, He parmi les épîtres pauliniennes, admettant ainsi une authenticité commandée en quelque sorte par le statut canonique désormais reconnu à l'écrit. ${ }^{39}$

A partir de là, l'authenticité ne sera plus remise en doute jusqu'à ce qu'Erasme renoue avec les objections, stylistiques notamment, des anciens et que l'exégèse moderne reconnaisse le bien fondé de ces objections.

Reste à prendre la question par l'autre bout.

Si l'on a rattaché He à la tradition paulinienne, était-on fondé à le faire, et, le cas échéant, jusqu'à quel point?

Nous procéderons ici en quatre temps en nous intéressant à quatre approches qui peuvent illustrer autant de facettes et d'orientations possibles de la recherche.

Les travaux de Ceslas Spicq représentent un premier type d'approche, dans la mesure où, convaincu qu'He « exprime sous des formules traditionnelles une pensée originale », il estime que « Paul a pu en être la source et l'inspirateur, mais qu'il ne pas lui-même rédigée ». ${ }^{40}$

Il reconnaît cependant des divergences: alors que Paul martèle qu'il a reçu l'Evangile directement de la part du Christ, l'auteur d'He se range parmi ceux qui [467] ont reçu cet Évangile par d'autres, qui l'ont eux-mêmes transmis (He 2,3) $;^{41}$ la christologie sacerdotale d'He «n'a pas de parallèle - ce qui ne veut pas dire d'attache ou d'amorce - dans l'œuvre de [...] Paul » ; ${ }^{42}$ l'écrit s'avère résolument théocentré alors que les épîtres pauliniennes sont, pour leur part, christocentrées $;^{43}$ l'auteur d'He évoque avec prédilection « le nom humain de Jésus » alors que Paul a une préférence marquée pour le Christ Jésus; il emploie à l'état absolu le titre «Fils" à son propos alors que le Tarsiote fait de même avec «Christ » $;^{44}$ le rôle alloué à l'Esprit Saint en He est très estompé par rapport à celui qui lui revient dans les épîtres pauliniennes $;{ }^{45}$ le motif de la justification est quasiment absent d'He ${ }^{46}$ et les oppositions entre «Loi et Evangile, lettre et esprit, esclavage et liberté, œuvres et foi » ne s'y trouvent carrément pas. ${ }^{47}$ D'ailleurs, constate Spicq, « pour Paul, c'est la Loi qui est à la base de toute l'économie mosaïque », alors que, « pour He, c'est le sacerdoce ${ }^{48}{ }^{48}$ Enfin, l'ekklèsia est une autre grande absente d'He. ${ }^{49}$

A ces différences qui touchent au fond, Spicq en ajoute d'autres, qui touchent à la forme. «Sur les neuf cent quatre-vingt-douze mots qui constitueraient le lexique de Hébr., deux cent

\footnotetext{
${ }^{37}$ Jérôme, Des hommes illustres 59. Il indique que Gaïus ne dénombre que treize épîtres de Paul et que, pour ce qui est la quatorzième, il dit qu'elle n'est pas de lui, considérée qu'elle est, chez les Romains, jusqu'à aujourd'hui comme n'étant pas de lui.

38 Jérôme, Lettre 139,3.

${ }^{39}$ Sur tout cela Spicq, Hébreux (note 1), 185-189.

${ }^{40}$ Spicq, Hébreux (note 1), 145. [467]

${ }^{41}$ Spicq, Hébreux (note 1), 145.

${ }^{42}$ Spicq, Hébreux (note 1), 146.

${ }^{43}$ Spicq, Hébreux (note 1), 146.

${ }^{44}$ Spicq, Hébreux (note 1), 147.

${ }^{45}$ Spicq, Hébreux (note 1), 147-148.

${ }^{46}$ Spicq, Hébreux (note 1), 149.

${ }^{47}$ Spicq, Hébreux (note 1), 150.

${ }^{48}$ Spicq, Hébreux (note 1), 150.

${ }^{49}$ Spicq, Hébreux (note 1), 151.
} 
quatre-vingt-douze ne se trouveraient pas dans [...] Paul $» ;^{50}$ la langue harmonieuse d'He, « la meilleure de tout le Nouveau Testament ${ }^{51}$, contraste avec « l'éloquence fougueuse de [...] Paul $»^{52}$; l'emploi qui est fait des particules en He est très différent de celui que l'on observe chez Paul, ${ }^{53}$ de même que la façon de citer l'Ecriture, non pas en tant qu'écriture mais en tant que parole. $^{54}$

Cela étant, Spicq signale aussi des ressemblances qui revêtent à ses yeux un grand poids. Si He comporte davantage d'hapax du Nouveau Testament que n'importe laquelle des épîtres pauliniennes - 152, nombre qu'il ramène à 57 en soustrayant les hapax qui se rencontrent dans les citations des LXX et les termes [468] que l'on y retrouve par ailleurs -, « un bon nombre correspondent au goût paulinien ». ${ }^{55}$ " He et [Paul] ont en commun » 65 mots «qui ne se rencontrent pas ailleurs dans le Nouveau Testament $\gg .{ }^{56}$ Spicq fait valoir encore une série de motifs théologiques communs, comme la présentation de la Passion sous l'angle d'une obéissance consciente (He 5,8; Rm 5,19; 2 Co 10,5; Ph 2,8), l'inefficacité de la Loi (He 10,1-4; Rm 3,20 ; 5,20; Ga 3,19) et son abrogation par le Christ, ${ }^{57}$ ainsi qu'une proximité entre la christologie d'He et celle des passages de nature hymnique que l'on trouve dans les épîtres de la captivité ( $\mathrm{Ph} \mathrm{2,6-10;} \mathrm{Col} \mathrm{1,15-18;} \mathrm{Ep} \mathrm{1,20-22),} \mathrm{avec} \mathrm{des} \mathrm{points}$ communs comme le Fils image de Dieu, son assimilation aux humains, son élévation audessus des anges et sa session à la droite de Dieu, ainsi que la réception d'un nom au-dessus de tout nom. ${ }^{58}$

Cette approche, souvent quasi statistique ou qui s'appuie sur des affirmations christologiques assez fondamentales, nous paraît masquer ou, en tout cas, ne pas prendre suffisamment en compte l'essentiel. Il y a manifestement des différences majeures entre $\mathrm{He}$ et les épîtres pauliniennes et il faut voir en He des développements largement originaux au sein du Nouveau Testament, même s'ils ne le sont jamais totalement.

Il y a tout d'abord le motif distinctif du Dieu qui parle, pour s'adresser le plus souvent au Fils qui très vite entre en dialogue avec lui pour endosser une mission dont les effets bénéfiques visent les destinataires $(2,12)$, des destinataires auxquels Dieu s'adresse aussi directement, si bien que c'est l'attitude de ces derniers, leur capacité de réception, d'écoute qui est en cause (voir surtout $3,7-4,11 ; 5,11-14 ; 12,25-29$ ) et qui est l'enjeu de la "parole d'exhortation » (He 13,22). Il ne suffit pas de signaler en note qu'en Rm 9,26 Paul introduit une citation scripturaire en faisant valoir que l'appel des païens est conforme à ce que Dieu dit en Osée $2,{ }^{59}$ pour atténuer cette différence marquante. Il nous semble d'ailleurs que l'on peut lire tout le sermon comme une sorte d'appel du Dieu qui parle, ${ }^{60}$ un appel relayé par le Fils et par tous ceux qui se sont mis à son écoute dans une chaine de la Parole qui mène jusqu'aux destinataires, appelés à leur tour à se mettre à l'écoute dans l'aujourd'hui de cet appel. La manière dont [469] est envisagée la venue du Fils en 5,7-10 pourrait d'ailleurs s'avérer

\footnotetext{
${ }^{50}$ Spicq, Hébreux (note 1), 152.

${ }^{51}$ Spicq, Hébreux (note 1), 152.

${ }^{5}$ Spicq, Hébreux (note 1), 153.

${ }^{53}$ Spicq, Hébreux (note 1), 154.

${ }^{54}$ Spicq, Hébreux (note 1), 154-155. [468]

${ }^{55}$ Spicq, Hébreux (note 1), 158-159.

${ }^{56}$ Spicq, Hébreux (note 1), 159-160.

${ }^{57}$ Spicq, Hébreux (note 1), 160-161.

${ }^{58}$ Spicq, Hébreux (note 1), 161-164.

${ }^{59}$ Spicq, Hébreux (note 1), n. 1, 155.

${ }^{60}$ Sur l'importance du motif du Dieu qui parle, voir notamment le recueil d'articles de Knut Backhaus, Der Sprechende Gott. Gesammelte Studien zum Hebräerbrief, WUNT 240 (Tübingen : Mohr Siebeck, 2009), 65-67, et Knut Backhaus, Der Hebräerbrief, RNT (Regensburg : Friedrich Pustet, 2009), passim. [469]
} 
exemplaire. L'attitude qui lui est attribuée peut faire penser au récit de la vigile à Géthsémané des évangiles. Toutefois, le vocabulaire employé est caractéristique des psaumes, qui sont, par ailleurs, les textes scripturaires les plus cités ou les plus prononcés tout au long du sermon, et le code de l'écoute est tout particulièrement présent, puisqu'il est indiqué que le Fils s'est exprimé à l'aide de prières, de supplications et à grand cri. Son attitude est caractérisée par

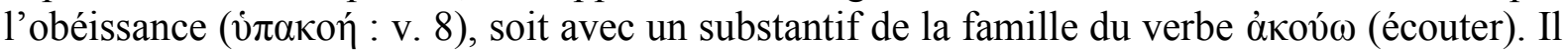

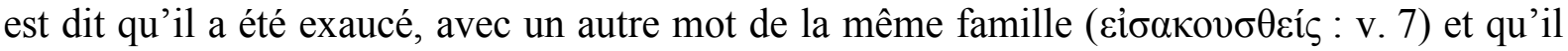

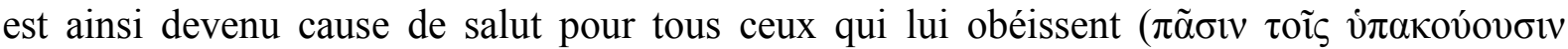
$\alpha \hat{\tau} \tilde{\omega}:$ v. 9).

La christologie sacerdotale, qui se déploie à l'échelle de l'œuvre tout entière, est un autre signe distinctif d'He. Certes, on peut trouver des indices d'une telle christologie ailleurs dans le Nouveau Testament, et notamment chez Lc, où le Ressuscité, prenant congé des disciples, les bénit à la manière du grand prêtre Onias en Si 50,20, ${ }^{61}$ mais nulle part ailleurs elle n'est filée avec une telle constance. De même, on peut trouver une amorce de la réinterprétation du

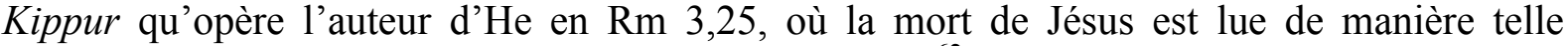
qu'elle apparaît récapituler les rites effectués ce jour-là. ${ }^{62}$ Cela étant, ce qui reste implicite en Rm 3,25 est longuement développé en He.

Quant à l'attitude par rapport à la Loi, si les conclusions de l'auteur d'He rejoignent dans leur radicalité celles d'un Paul $(\mathrm{He} 7,18)$, son argumentation est très différente. Il souligne en effet le lien indéfectible et réciproque existant entre le sacerdoce ancien et la Loi de Moïse (He 7,11 et 12). Pour lui, qui s'intéresse d'abord à la médiation, la Loi est au premier chef, tout comme le sacerdoce, une institution de médiation. Et la partie essentielle de cette Loi est précisément celle qui vise à assurer une relation harmonieuse, la rencontre, la communion entre le peuple et Dieu à travers l'institution du sacerdoce. Son efficacité doit donc être jaugée à l'aune de celle du sacerdoce dont elle régit le fonctionnement.

L'auteur d'He développe donc une compréhension cultuelle de la Loi. [470]

Paul, de son côté, en a développé d'abord une compréhension éthique, refusant le postulat selon lequel l'économie du faire $(\mathrm{Rm} 7,7-8,4 ; 10,5-6)$ confèrerait à l'homme sa justification. Selon lui, l'Evangile réside d'abord dans la proclamation de l'événement de salut qui se concentre dans la Croix et la Résurrection (1 Co 15,1-5). C'est à partir de cette proclamation de foi qu'il a été amené à dissocier Loi et grâce et à refuser toute fonction sotériologique à la Loi pour développer au contraire sa fonction accusatrice. Cela ne l'a pas empêché toutefois, et le point est essentiel, de maintenir l'exigence éthique et de l'exprimer notamment autour du thème de l'accomplissement (et non plus du faire) de la Loi (Ga 5,4;6,2; Rm 8,4;13,8-10). Cet accomplissement de la Loi, envisagé comme un fruit de l'Esprit au sein de l'économie nouvelle de la justification par la foi, passe par une insistance sur le commandement d'amour ( Rm 13,8.10) et s'accompagne d'une abrogation de la Loi rituelle, même si les forts peuvent être amenés à s'y plier par respect pour les faibles (Rm 14,14ss).

On pourra dire que, aux yeux de Paul, la Loi est disqualifiée parce qu'elle ne peut pas donner la vie ou conférer la justification, alors que, pour l'auteur d'He, elle est disqualifiée parce

\footnotetext{
${ }^{61}$ Nous avons développé ce point dans notre contribution « De Zacharie à Jésus ressuscité, la construction de la figure du prêtre de par et d'autres de l'Evangile selon Luc et au début des Actes », in Camille Focant et André Wénin, éd., Analyse narrative et Bible. Deuxième colloque international du RRENAB, Louvain-La-Neuve, avril 2004, BEThL 191 (Leuven : University Press, 2005), 297-308.

${ }^{62}$ Voir notamment sur ce point, Wolfgang Kraus, Der Tod Jesu als Heiligtumsweihe. Eine Untersuchung zum Umfeld der Sühnevorstellung in Römer 3,25-26a, WMANT 66 (Neukirchen: Neukirchener Verlag, 1991), plus particulièrement 235-259. [470]
} 
qu'elle ne peut pas apporter la $\tau \varepsilon \lambda \varepsilon i ́ \omega \sigma ı \varsigma .{ }^{63}$ Ou encore, pour Paul, la Loi est inefficace parce que les hommes ne la font pas ; pour He, elle est inefficace parce que ce ne sont que des êtres humains qui l'accomplissent. ${ }^{64}$

Tout cela montre l'écart qui sépare He des écrits pauliniens, même si la christologie et la connaissance de traditions qui circulaient au sein du mouvement chrétien naissant rendent l'existence de points communs tout à fait compréhensible.

Un deuxième type d'explication est fourni par Clare K. Rothschild. ${ }^{65}$ Elle propose, quant à elle, que l'auteur, inconnu, d'He ait d'emblée et consciemment recouru à l'imitation du style de Paul et à l'emprunt d'expressions de ses lettres pour faire passer son œuvre et ses vues souvent radicales pour pauliniennes, et contribuer plus encore à universaliser la portée du corpus paulinien en le pourvoyant d'un discours adressé par l'apôtre aux juifs.

Selon elle, l'imitation de Paul ne se limite pas à He 13,20-25, conclusion de l'écrit au sein de laquelle le nombre de correspondances avec le corpus paulinien est particulièrement frappant comme le tableau que nous proposerons plus loin le fait apparaître. Elle estime que l'imitation consciente de Paul transparaît [471] en fait tout au long de l'œuvre et que " d'autres passages d'He démontrent aussi une dépendance par rapport aux lettres de Paul $\gg .{ }^{66}$

Le défaut de la démonstration que mène alors Rothschild réside, selon nous, dans le fait qu'elle privilégie trop exclusivement les parallèles que l'on peut effectuer avec la littérature paulinienne et qu'elle ne tient pas suffisamment compte de travaux qui, tels ceux de Spicq, ont montré que les parallèles ne se limitent nullement à la littérature paulinienne mais affectent de nombreux autres écrits, qu'il s'agisse notamment de la littérature johannique ou de 1 Pierre. ${ }^{67}$ Sans les négliger, elle nous parait trop les relativiser en mettant en avant l'argument selon lequel l'origine des motifs que l'on rencontre ailleurs au sein des épîtres, canoniques ou non, se trouve chez Paul, ${ }^{68}$ ce qui relève de l'évidence et ne prouve rien.

Elle veut aussi que les citations scripturaires que l'on rencontre en Hébreux se retrouvent aussi chez Paul, mais elle mène son enquête à l'échelle des chapitres cités et non pas seulement des versets concernés et arrive ainsi à 17 correspondances ${ }^{69}$ au lieu de $9 .{ }^{70}$

Les huit correspondances qui se limitent à la citation d'un même chapitre sont regroupées dans le tableau suivant :

\begin{tabular}{|c|c|c|}
\hline Chapitre concerné & Citation(s) chez Paul (et verset concerné) & Citation(s) en He (et verset concerné) \\
\hline Gn 2 & 1 Co 15,45 (v. 7); 1 Co 6,16 (v. 24) & He 4,4 (v. 2) \\
\hline Gn 22 & Ga $3,8.16(\mathrm{v} .18)$ & He 6,13-14 (vv. 16-17) ; He 11,12 (vv. 17) \\
\hline Dt 17 & 1 Co 5,13 (v. 7) & He 10,28 (v. 6 ) \\
\hline Dt 29 & Rm 11,8 (v. 4) & He 12,15 (v. 18) \\
\hline Dt 32 & $\begin{array}{l}1 \text { Co } 10,20 \text { (v. } 17) ; \\
\operatorname{Rm} 10,19 \text { et } 1 \text { Co } 10,22 \text { (v. } 21)\end{array}$ & He 10,30 (v. 36) \\
\hline Pr 3 & $2 \operatorname{Co} 8,21$ (v. 4) & He $12,5-6$ (vv. 11-12) \\
\hline Es 8 & $\operatorname{Rm} 9,33$ (v. 14) & He 2,13 (vv. 17-18) \\
\hline Es 53 & Rm 10,16 (v. 1) & He 9,28 (v. 12). \\
\hline
\end{tabular}

\footnotetext{
${ }^{63}$ Harold W. Attridge, The Epistle to the Hebrews. A Commentary on the Epistle to the Hebrews, Hermeneia (Philadelphia : Fortress Press, 1989), 204.

${ }^{64}$ Ainsi Walter Gudbrod, « vó $\mu$ os », TDNT 4, 1022-1091, ici, 1079.

${ }^{65}$ Rothschild, Hebrews (note 36). [471]

${ }^{66}$ Rothschild, Hebrews (note 36), 81.

${ }^{67}$ Voir supra, note 1.

${ }^{68}$ Rothschild, Hebrews (note 36), 117.

${ }^{69}$ Rothschild, Hebrews (note 36), 90.

${ }^{70}$ Rothschild, Hebrews (note 36), 90. [472]
} 
Quant aux neuf correspondances qui touchent un même verset, elles sont regroupées ci-après : [472]

\begin{tabular}{|c|c|c|c|}
\hline $\begin{array}{l}\text { Verset } \\
\text { concerné }\end{array}$ & $\begin{array}{l}\text { Citation chez } \\
\text { Paul }\end{array}$ & Citation en Hébreux & Caractère de la correspondance \\
\hline Gn 15,5 & $\operatorname{Rm~4,18}$ & He 11,12 & $\begin{array}{l}\text { En He, allusion plutôt que citation portant sur une autre } \\
\text { partie du verset }\end{array}$ \\
\hline Gn 21,12 & $\operatorname{Rm} 9,7$ & He 11,18 & $\begin{array}{l}\text { Correspondance affectant un verset qui ne joue pas un } \\
\text { rôle majeur dans l'argumentation d'He }\end{array}$ \\
\hline Dt 32,35 & Rm 12,19 & He $10,30 \mathrm{a}$ & $\begin{array}{l}\text { Correspondance affectant un verset qui ne joue pas un } \\
\text { rôle majeur dans l'argumentation d'He }\end{array}$ \\
\hline Dt 32,43 & $\mathrm{Rm} 15,10$ & He 1,6 & Citation ne portant pas sur le même segment du verset \\
\hline $2 \mathrm{~S} 7,14$ & 2 Co $6,18 \mathrm{a}$ & He 1,5 & Passage cité également en Ap 21,7 \\
\hline Ps $8,5-7$ & $1 \mathrm{Co} 15,27$ & He $2,6-8$ & $\begin{array}{l}\text { Correspondance réelle, touchant un passage } \\
\text { particulièrement important dans l'argumentnation d'He }\end{array}$ \\
\hline Ps $109,1 \mathrm{LXX}$ & $1 \mathrm{Co} 15,25$ & $\begin{array}{l}\mathrm{He} 1,3.13 ; 8,1 ; \\
10,12-13 ; 12,2\end{array}$ & $\begin{array}{l}\text { Correspondance réelle, touchant un passage } \\
\text { particulièrement important dans l'argumentnation d'He }\end{array}$ \\
\hline Jr 31,33-34 & $\operatorname{Rm} 11,27$ & $\begin{array}{l}\mathrm{He} 8,8-12 ; 10,16- \\
17\end{array}$ & Correspondance qui n'est nuellment évidente \\
\hline Ha $2,[3]-4$ & $\begin{array}{l}\text { Rm } 1,17 ; \\
\text { Ga } 3,11\end{array}$ & He $10,37-38$ & $\begin{array}{l}\text { Correspondance affectant un verset qui ne joue pas un } \\
\text { rôle majeur dans l'argumentation d'He }\end{array}$ \\
\hline
\end{tabular}

Si l'on se penche sur ces 9 correspondances, qui seules peuvent entrer en ligne de compte, on constate que certaines ne sont pas réelles. C'est ainsi que l'une concerne une allusion plutôt qu'une citation, allusion qui, de plus, n'a pas directement trait au passage qui est cité chez Paul, ${ }^{71}$ qu'une autre affecte un segment de verset qui n'est pas le même que celui qui est cité en $\mathrm{He}^{72}$ et qu'une troisième est tout sauf évidente puisqu'il faudrait admettre que la reprise

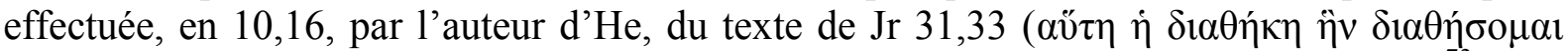

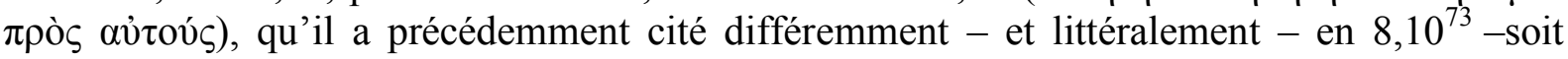
inspirée par Rm 11,27, qui n'est pas une citation de Jr 31,31-34, mais de Jr 59,20-21, et qui comporte, quant à elle, le pronom personnel à la troisième [473] personne du pluriel employé

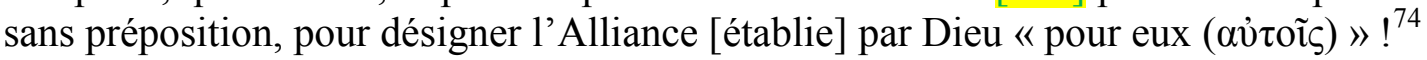

Des neuf correspondances alléguées, il n'en reste plus que six.

Sur ces six correspondances, deux seulement touchent des passages qui s'avèrent plus particulièrement importants dans l'argumentation de l'auteur, cela dans la mesure où ils font l'objet d'un commentaire subséquent de sa part. Il s'agit de Ps 8,7, qui est cité tant en 1 Co 15,27 qu'en Ep 1,22, mais plus brièvement qu'en He 2,6-8 où ce sont les versets 5-7 sont cités, et du Ps 109,1 (LXX), qui est cité en 1 Co 15,25, mais l'est aussi en Mc 12,36 et // et en Ac 2,34. En revanche, ni le Ps 2,7 (LXX), qui est cité à deux reprises en He 1,5a et en He 5,5, et qui apparaît à l'arrière-plan du message de la voix céleste dans les récits synoptiques du

\footnotetext{
${ }^{71} \mathrm{Rm} 4,18$ et He 11,12 (Gn 15,5). Rothschild, Hebrews (note 36), 94-95, en fait cependant grand cas.

${ }^{72} \mathrm{Rm} \mathrm{15,10}$ et He 1,6 (Dt 32,43). Rothschild, Hebrews (note 36), 97-98, tout en reconnaissant l'apparente absence de relation intertextuelle, maintient pourtant là aussi que la citation du même passage - il faudrait dire du même verset, sans que ce soit de la même partie du verset - renforce aux yeux de lecteur ayant lu Rm - le caractère paulinien d'He.

${ }^{73}$ Dans un premier temps, il a été indiqué que l'Alliance a été établie «pour la maison d'Israël $(\tau \tilde{\omega}$ oốk

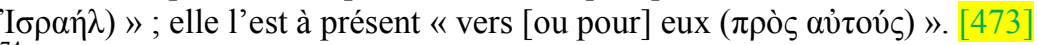

${ }^{74}$ Pour mesurer la difficulté de l'hypothèse, on précisera qu'il faudrait que l'auteur d'He soit passé d'une construction dans laquelle $\delta 1 \alpha \theta \eta ́ \sigma o \mu \alpha$ était suivi d'un complément d'attribution au datif non précédé d'une

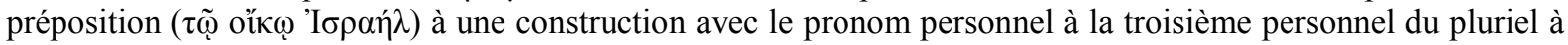
l'accusatif et précédé d'une préposition ( $\pi \rho o ́ \varsigma \alpha v ่ \tau o v ́ \varsigma)$, cela sous l'influence d'un texte qui est lui-même construit avec le pronom personnel à la troisième personne du pluriel au datif seul !
} 
baptême de Jésus (Mc 1,11 et $/ /)^{75}$ ainsi que comme parole accompagnant l'exaltation de Jésus ressuscité en Ac 13,33, ni le Ps 95,7-11, cité en He 3,7b-11, puis repris par bribes en $\mathrm{He}$ en 3,$15 ; 4,3.5 .7$ et longuement commenté jusqu'en 4,11, ni le Ps 110,4 , cité à trois reprises en He 5,6 et 7,17.21, ni Gn 14,17-20, qui est utilisé en fonction d'un soigneux découpage en $\mathrm{He}$ 7,1-2 et qui se trouve au cœur de l'argumentation de la suite du chapitre, ni le Ps 40,7-9, cité en $\mathrm{He} 10,5-7$, puis repris et commenté dans les versets qui suivent (He 10,9-10), ne sont produits dans les épîtres pauliniennes.

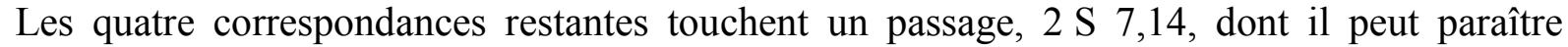
problématique de dire, au sens strict, qu'il n'est cité que par Paul, dans la mesure où il l'est également en Ap 21,7, ${ }^{76}$ et des versets qui ne jouent pas un rôle aussi important dans l'argumentation que ceux dont nous avons souligné le rôle majeur précédemment, qu'il s'agisse de Dt 32,35, cité en He 10,30a [474] et Rm 12,19, d'Ha 2,[3-]4, cité, d'une part, en He 10,37-38 et, d'autre part, en Rm 1,17 et en Ga 3,11, ${ }^{77}$ et enfin de Gn 21,12 (LXX), cité en He 11,18 et Rm 9,7.

Quand on mesure par ailleurs l'importance du fait que les citations scripturaires sont systématiquement introduites par l'auteur d'Hébreux en tant que paroles et non pas en tant qu'Ecriture, ${ }^{78}$ on conviendra que les arguments de Rothschild peuvent peiner ici à emporter l'adhésion quand on réexamine minutieusement le dossier.

Le balayage qu'elle effectue, en 10 pages, ${ }^{79}$ des chapitres 1 à 12 pour faire valoir qu'ils présentent des points communs avec les épîtres pauliniennes, ce dont personne ne doute, s'avère trop rapide et trop unilatéral pour s'avérer convaincant, en fonction précisément des divergences qui demeurent sur un plan plus fondamental entre Hébreux et le corpus paulinien et des ressemblances que l'on peut aussi discerner avec d'autres écrits du Nouveau Testament. ${ }^{80}$

Rothschild, s'appuyant notamment sur le témoignage du $\mathrm{P}^{46}$, sur lequel nous aurons à revenir et qui, au sein de la tradition manuscrite, est le plus ancien témoin de l'œuvre (vers 200) et l'incorpore entre Romains et 1 Corinthiens dans ce qui apparait comme une collection d'épîtres pauliniennes, estime encore que l'auteur serait parvenu à ses fins. ${ }^{81}$ Il aurait déployé avec succès une rhétorique de l'allusion, proposant une nouvelle révélation sur la base de l'ancienne, et aurait réussi au point que son œuvre resterait associée au corpus paulinien et serait très majoritairement attribuée au Tarsiote, du moins jusqu'à la Réforme. Il n'en demeurerait pas moins que l'écrit, en invitant les destinataires à passer d'un enseignement certes correct, mais préliminaire, aux choses véritablement solides $(6,1-2)$, recadrerait ou reprendrait consciemment certains enseignements de Paul, tels ceux qui ont trait à la

\footnotetext{
${ }^{75}$ Il est même tout à fait possible qu'il ait constitué l'intégralité dudit message dans le troisième évangile si la leçon attestée en Lc 3,22 par le codex de Bèze (D), les vieilles versions latines, Justin, Méthode, Hilaire et

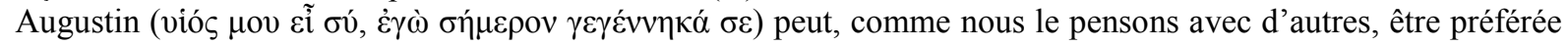
à la leçon retenue par les éditeurs du Nestle-Aland, leçon qui s'aligne, quant à elle, sur les parallèles de Marc et de Matthieu et qui peut passer pour une harmonisation sans pouvoir prétendre être non plus ni lectio difficilior ni mère des variantes.

${ }^{76}$ Pour Rothschild, Hebrews (note 36), 98, la citation ne ferait que renforcer l'impression qu'He est de Paul (98), cela alors même qu'elle se trouve au tout début de l'écrit et que celui-ci commence fort différemment des épîtres pauliniennes ! [474]

${ }^{77}$ On notera toutefois que le découpage est différent et, surtout, que la perspective n'est pas la même : Ha 2,4 joue un rôle décisif chez Paul, alors qu'on ne peut affirmer la même chose pour ce qui est d'Hébreux.

${ }^{78}$ Ces citations sont introduites le plus souvent par $\lambda \dot{\varepsilon} \gamma \omega(\mathrm{He} 1,5.6 .7 .13 ; 2,6.12 ; 3,7.15 ; 4,3.4 .7 ; 5,6 ; 6,13$; $7,21 ; 8,8 ; 9,20 ; 10,5.8 .9 .30 ; 12,21.26 ; 13,5.6)$ et encore par $\pi \rho 0 \lambda \varepsilon ́ \gamma \omega(\operatorname{He} 4,7), \lambda \alpha \lambda \varepsilon ́ \omega(\operatorname{He} 5,5 ; 11,18)$,

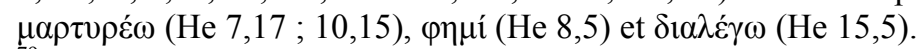

${ }^{79}$ Rothschild, Hebrews (note 36), 106-116.

${ }^{80}$ Voir supra, note 1, où sont indiqués les chapitres que Ceslas Spicq consacre à la question.

${ }^{81}$ Rothschild, Hebrews (note 36), 142-144. [475]
} 
résurrection des morts, dont les limites apparaissaient à l'auteur sur le plan historique. Ils seraient réintréprétés ici dans le cadre d'une alliance qui existe indépendamment de l'espace et du temps et [475] qui n'est donc pas exposée aux limitations de l'histoire, cela pour compléter et réorienter une première collection de lettres de Paul. ${ }^{82}$

Comme nous l'avons dit, l'argumentation pour habile et riche qu'elle soit, ne nous paraît pas convaincante pour autant.

Il nous semble qu'il convient en fait de partir du passage derrière lequel transparait la figure de Paul, à savoir la finale de l'écrit, qui, en citant notamment la figure bien connue du frère et compagnon de route Timothée, désormais relâché, donne au lecteur l'impression que c'est Paul en personne qui s'apprête à venir rendre visite aux destinataires en sa compagnie (He 13,23). Il est notoire que de nombreux parallèles peuvent être établis entre cette finale et celle d'autres épîtres néotestamentaires, plus particulièrement pauliniennes, et ce sont ces parallèles dont il conviendra de prendre la mesure avant même d'étudier les deux derniers types de réponses qui ont été apportées à la question du rapport d'He à la tradition paulinienne.

Nous étendrons, pour des raisons qui appraîtront plus loin, l'état des lieux à l'échelle d'He 13,18-25 en prenant en compte à la fois des parallèles que ces versets présentent avec le corps de l'écrit et avec d'autres épîtres du Nouveau Testament, tout particulièrement dans leurs sections finales.

\begin{tabular}{|c|c|c|}
\hline Texte d'Hébreux & Verset & $\begin{array}{l}\text { Parallèles néotestamentaires (plus particulièrement dans le } \\
\text { cadre des finales épistolaires) }\end{array}$ \\
\hline 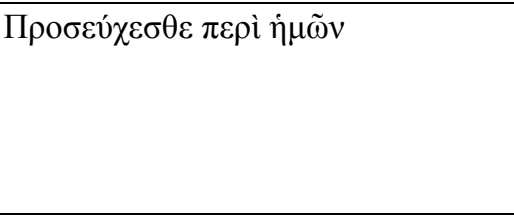 & 13,18 & 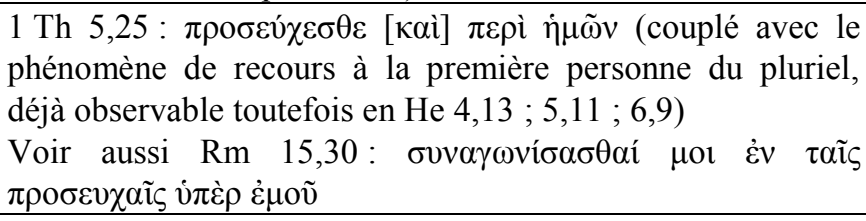 \\
\hline $\begin{array}{l}\pi \alpha \rho \alpha \kappa \alpha \lambda \tilde{\omega} \tau o \tilde{\tau} \tau \text { o } \pi \text { oiñ } \sigma \alpha l \\
\text { (à propos de la prière) }\end{array}$ & 13,19 & 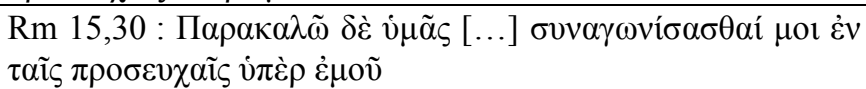 \\
\hline 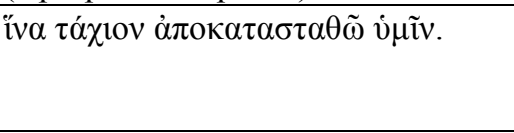 & 13,19 & 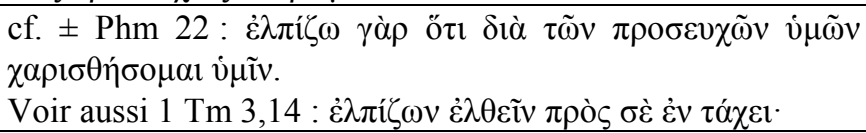 \\
\hline 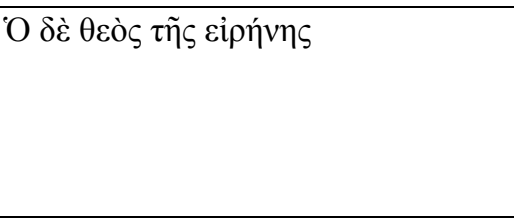 & 13,20 & 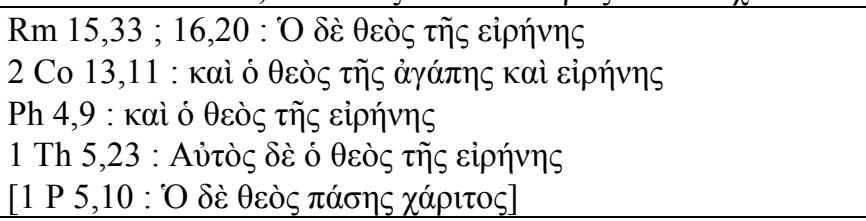 \\
\hline 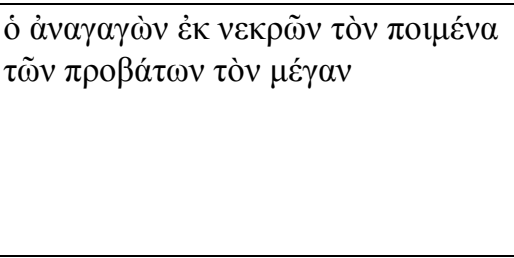 & 13,20 & 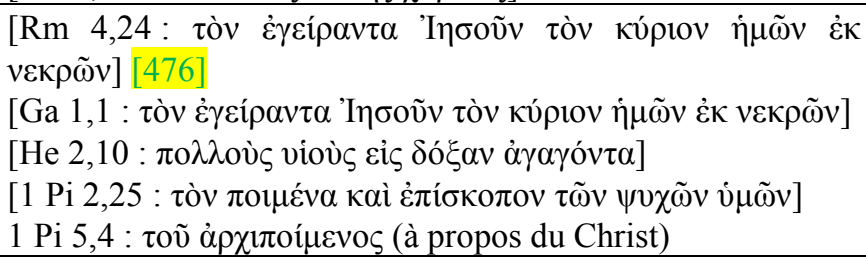 \\
\hline 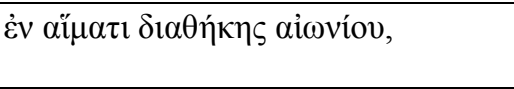 & 13,20 & 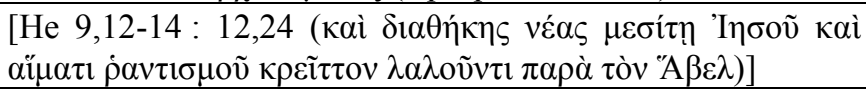 \\
\hline 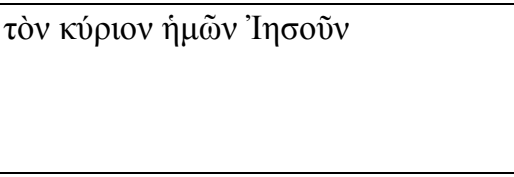 & 13,20 & 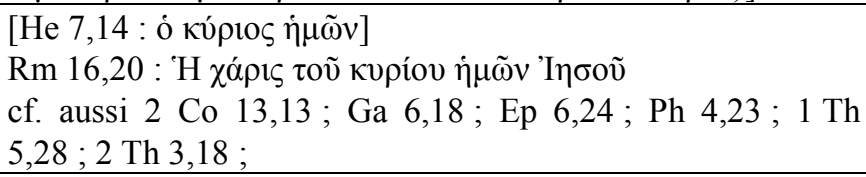 \\
\hline 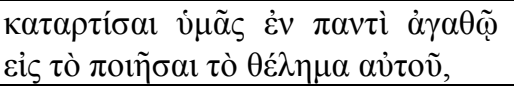 & 13,21 & 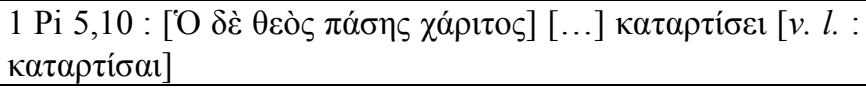 \\
\hline 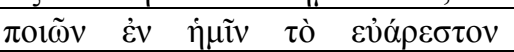 & 13,21 & \\
\hline
\end{tabular}

\footnotetext{
${ }^{82}$ Voir notamment Rothschild, Hebrews (note 36), 206. [477]
} 


\begin{tabular}{|c|c|c|}
\hline 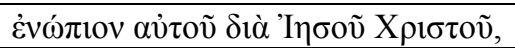 & & 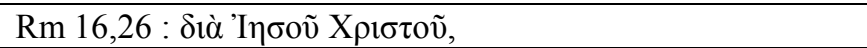 \\
\hline 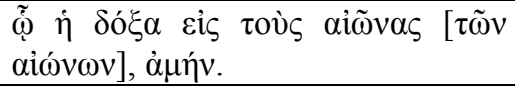 & 13,21 & 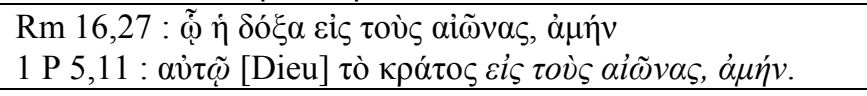 \\
\hline 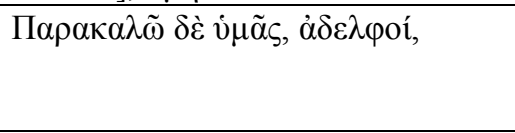 & 13,22 & 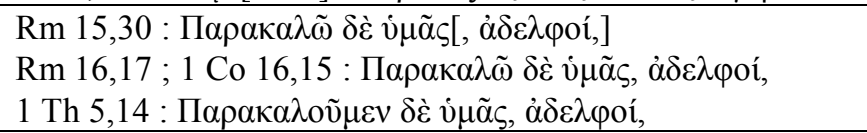 \\
\hline 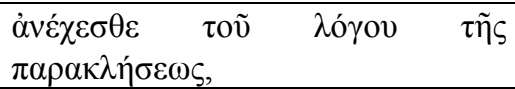 & 13,22 & 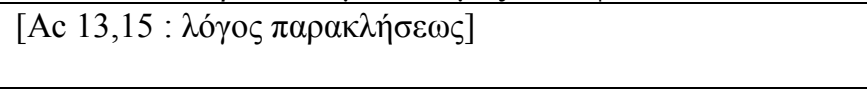 \\
\hline 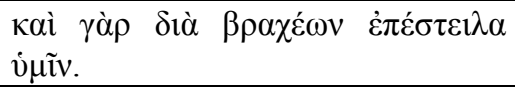 & 13,22 & 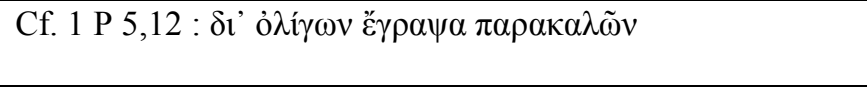 \\
\hline 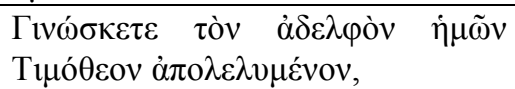 & 13,23 & 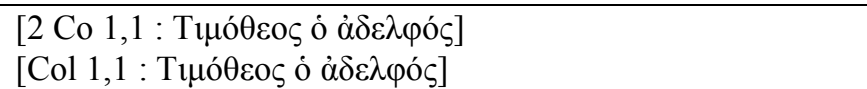 \\
\hline 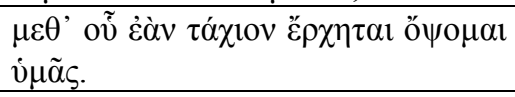 & 13,23 & \\
\hline 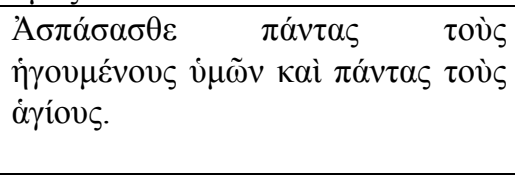 & 13,24 & 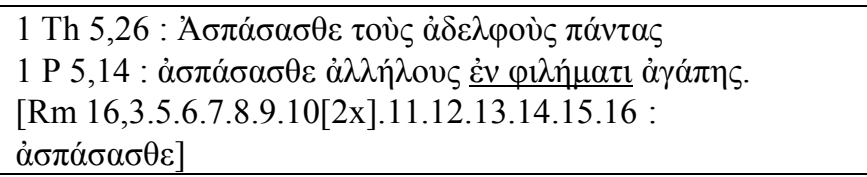 \\
\hline 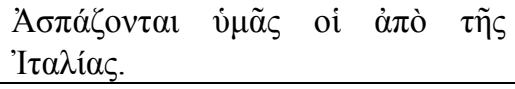 & 13,24 & 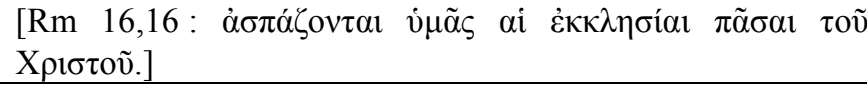 \\
\hline 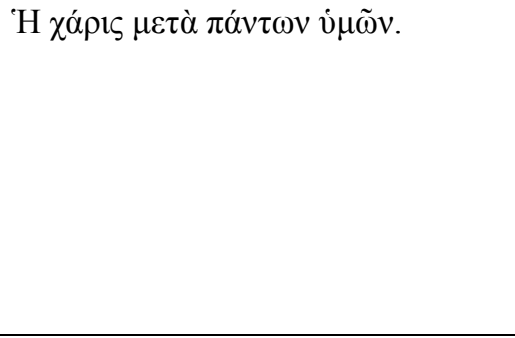 & 13,25 & 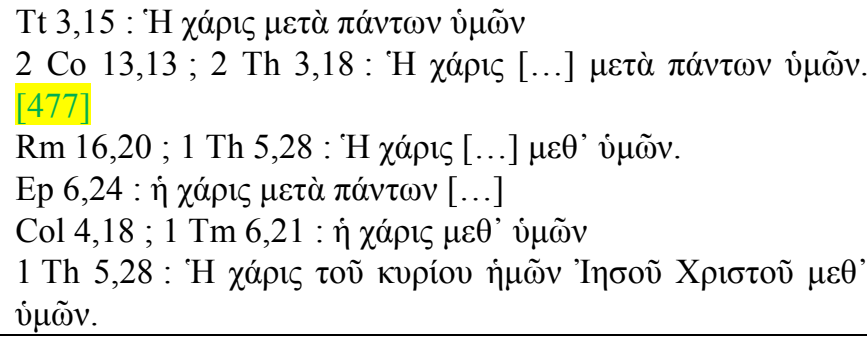 \\
\hline
\end{tabular}

En fonction de ces parallèles fort impressionnants, un premier type d'explications - qui sera pour nous le troisième en ce qui concerne la question des rapports entre $\mathrm{He}$ et la tradition paulinienne - est représenté par une étude très poussée de Knut Backhaus dans une contribution portant précisément sur la question du rapport entre He et l'école paulinienne, ${ }^{83}$ contribution qu'il a reprise pour l'essentiel dans son récent commentaire d'He. ${ }^{84}$

Il part de la bénédiction (He 13,20-21) et des paroles d'envoi finales (He 13,22-25) et estime, de manière parfaitement fondée nous semble-t-il, que c'est à partir de cette conclusion à caractère épistolaire que l'on est le mieux à même de rendre compte des rapports qu'entretient He avec la tradition paulienne. Cela étant, il considère que ces paroles d'envoi appartenaient déjà à l'écrit primitif, ce qui distingue son approche de la dernière de celles que nous aborderons. Il défend, dès lors, la thèse suivante: «He 13,22-25 permet de conclure à l'existence de liens sociaux et théologiques concrets entre la communauté [destinataire] d'Hébreux - qui aurait été une communauté domestique de la capitale de l'Empire - et l'école paulinienne romaine $\gg$. $^{85}$

Il fait observer que ce que nous savons ou pouvons supposer des communautés romaines à la fin du premier siècle de notre ère cadre fort bien avec le portrait que l'on peut dresser des destinataires implicites à la lumière de l'écrit: présence vraisemblable de plusieurs communautés domestiques dans la cité $(\mathrm{He} 13,24)$; existence d'une catéchèse déjà développée en leur sein ; connaissance de la figure de Timothée, mentionnée en He 13,23

\footnotetext{
${ }^{83}$ Knut Backhaus, « Der Hebräerbrief und die Paulus-Schule », BZ 37 (1993) : 183-208, repris in Backhaus, Der Sprechende Gott (note 60), 21-48.

${ }^{84}$ Backhaus, Hebräerbrief (note 60), 23-26.

${ }^{85}$ Backhaus, Der Sprechende Gott (note 60), 42. [478]
} 
comme en Rm 16,21 ; ancienneté des persécutions (He 10,32-34) conduites à l'encontre des chrétiens dans la capitale de l'Empire ; dominante judéo-chrétienne au sein de la chrétienté ro[478]maine au moins jusqu'à l'édit de Claude, ce qui permet de rendre compte de la familiarité avec l'arrière-plan scripturaire que suppose l'écrit. ${ }^{86}$

Par ailleurs, la salutation de la part de ceux qui proviennent d'Italie se comprend mieux si l'écrit est précisément adressé à des destinataires qui se trouvent dans ce pays ${ }^{87}$, et He est connue très anciennement à Rome, ce qu'atteste 1 Clément. A cela s'ajoute le fait que le titre (pro)hègoumenoi n'est attesté, pour les responsables de la communauté, qu'à Rome (1 Clém. 1,$3 ; 21,6 ;{ }^{88}$ Hermas, Pasteur, vision 2,2,6;3,9,7 $7^{89}$ ), en dehors d'He 13,7.17.24 où revient à trois reprises hègoumenoi. ${ }^{90}$

Backhaus propose ainsi que les destinataires soient en fait une communauté de maison, composée de membres à la fois cultivés, plutôt aisés et d'un niveau intellectuel élevé, intéressés à comprendre leur appartenance chrétienne à la lumière de l'arrière-plan fourni par le judaïsme. Ce public, présent dans la capitale de l'Empire, aurait tout naturellement pu être en contact avec des cercles pauliniens mais aussi avec les milieux à l'origine de 1 Pierre, écrite elle-même de Rome, reconnaissable sous le nom de code «Babylone " en 1 P 5,13. L'auteur et les destinataires auraient ainsi été au fait des conventions épistolaires en vigueur dans la mouvance paulinienne et de matériaux traditionnels exploités par la tradition paulinienne et par 1 Pierre et qu'Hébreux réinterprète à sa façon ${ }^{91}$.

Une autre voie, ouverte dès la fin $\mathrm{du} \mathrm{XVIII}^{\mathrm{e}}$ siècle et épisodiquement évoquée - mais aussi précisée - depuis, nous paraît être tout aussi astucieuse et finalement plus convaincante, même si elle demeure, elle aussi, indémontrable.

Elle consiste à reconnaître que He 13,22-25 à tout le moins - on verra que certains seraient tentés d'y adjoindre He 13,19 ou He 13,18-21 - constitue une addition secondaire et que le rattachement à la tradition paulinienne s'est donc effectué dans un deuxième temps.

Le premier, à notre connaissance, à avoir envisagé qu'He 13,22-25 puisse constituer un ajout est Johann Gottfried Immanuel Berger, qui fut répétiteur à la Faculté de Théologie de Göttingen et mourut à l'âge de 30 ans. ${ }^{92}$ Dès 1797, il a proposé qu'He 13,22-25 soit une addition, imputable à l'auteur lui-même ou à [479] un autre que lui. ${ }^{93}$ Franz Overbeck a effectué un pas de plus en faisant valoir que c'est pour placer l'épître sous l'autorité de Paul que la suscription et la finale ont été ajoutées. ${ }^{94}$ Se situant explicitement dans son sillage, William Wrede a défendu, pour sa part, les deux thèses suivantes : 1) He n'est en aucun cas

\footnotetext{
${ }^{86}$ Backhaus, Hebräerbrief (note 60), 25.

${ }^{87}$ Backhaus, Hebräerbrief (note 60), 25.

${ }^{88}$ Les deux titres ègoumenoi et proègoumenoi sont ici employés successivement.

${ }^{89}$ Proègoumenoi est utilisé dans les deux cas.

${ }^{90}$ Backhaus, Hebräerbrief (note 60), 25.

${ }^{91}$ Backhaus, Hebräerbrief (note 60), 25-26.

${ }^{92}$ On pourra se reporter sur le personnage à la notice suivante, disponible en ligne et qui précise qu'il signait ses œuvres en ne faisant pas toujours figurer l'intégralité de ses prénoms: http://kalliopeverbund.info/de/eac?eac.id=116131705 (notice consultée le 15 janvier 2017). [479]

${ }^{93}$ [Johann Gottfried] I[mmanuel] Berger, Der Brief an die Hebräer, eine Homilie, in Göttingische Bibliothek der neuesten theologischen Literatur. hg. von Joh. Friedr. Schleusner und Carl Friedr. Stäudlin. Dritter Band (Göttingen : Vandenhoeck \& Ruprecht, 1797), 449-469 (ici : 455) : Allein dieser Schluß [He 13,22-25] ist von der vorhergehenden Abhandlung so wohl durch seinen Inhalt als durch das vorgehende ḋǘv so sorgfältig abgesondert, daß man offenbar sieht, er gehöre nicht unmittelbar dazu. Es läßt sich daher die Schwierigkeit sehr gut dadurch heben, wenn man annimmt, die Rede sei entweder von ihrem Verfasser selbst, der sie, nachdem er sie gehalten hatte, aufgesezt habe, oder auch von einem andern, der sie entweder nachgeschrieben, oder ihre Gedanken so gut gefaßt hatte, daß er sie nachher aufsezen [sic] konnte, an irgend eine auswärtige Gemeine [sic], oder auch an mehrere geschikt [sic] worden].

${ }^{94}$ Franz Overbeck, Zur Geschichte des Kanons. Zwei Abhandlungen (Chemnitz: Ernst Schmeitzner, 1880$), 16$.
} 
une véritable lettre dirigée vers un public bien défini ; 2) par sa finale, He veut se donner malgré tout comme une épître de Paul et plus particulièrement comme une épître de la captivité. ${ }^{95}$ Dans son commentaire d'Hébreux, Erich Grässer est encore plus net et affirme qu'He 13,22-25 ne peut être un ajout de l'auteur d'Hébreux et qu'il s'agit en fait d'un postscriptum imputable à une main étrangère et destiné à conférer à l'écrit une dignité apostolique, opération qui a pleinement réussi dans la mesure où l'œuvre a fini par trouver sa place au sein du canon. ${ }^{96}$

Avant lui, Albert Vanhoye avait proposé un scénario distinguant deux éditions successives de l'écrit : " $1^{\circ}$ Le premier ouvrage est une longue prédication, composée avec application, destinée à être prononcée oralement, et qui fut prononcée de fait dans des communautés chrétiennes. Elle s'étend de l'exorde jusqu'à la première conclusion, c'est-à-dire 13,21, à $l^{\prime}$ exception de $13,19.2^{\circ}$ Le second ouvrage est une brève addition, d'un style hâtif, ajoutée lorsque la [480] prédication fut envoyée à une autre communauté. Cette lettre consiste en une phrase insérée avant la péroraison $[13,19]$ et quelques autres ajoutées après la péroraison $[13,22-25]$. Elle contient des nouvelles et des salutations. ${ }^{97}$

A l'appui de cette hypothèse, Vanhoye fait valoir que ces versets ont ceci « de commun qu'ils ne font pas vraiment corps avec l'ensemble du discours $»,{ }^{98}$ et cela même si un lien peut être établi, ce qui atteste que l'addition a été faite par un auteur qui avait pris la peine de prendre connaissance du discours dans son ensemble : la demande d'He 13,19 s'articule sur celle d'He 13,18; des éléments de vocabulaire sont repris, comme la mention des dirigeants (oi ทे discours d'exhortation ( $\lambda$ ó $\sigma \varsigma \varsigma \tau \tilde{\eta} \varsigma \pi \alpha \rho \alpha \kappa \lambda \eta ́ \sigma \varepsilon \omega \varsigma)$. En dépit des liens que l'on peut tisser avec le discours qui précède, Vanhoye fait valoir que ces cinq versets s'en distinguent clairement dans la mesure où ce sont les seuls, en dehors d'He 11,32, dans lesquels, au sein de l'écrit tel qu'il nous est parvenu, la première personne du singulier est employée en lieu et place de la

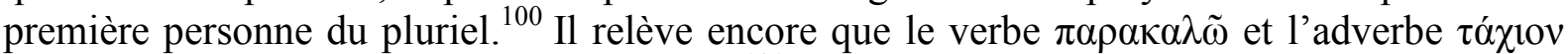
se retrouvent à la fois en $\mathrm{He} 13,19$ et $13,22^{101}$ et qu'He 13,22-25 relève manifestement d'un autre genre littéraire que l'ensemble du discours, celui du « billet d'envoi ». ${ }^{102}$ Le recours à ce

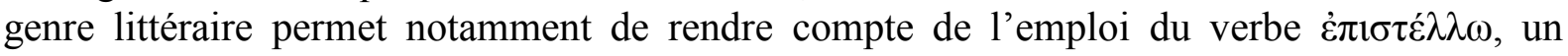

\footnotetext{
95 William Wrede, Das literarische Rätsel des Hebräerbriefs. Mit einem Anhang über den literarischen Charakter des Barnabasbriefes, FRLANT 8 (Göttingen : Vandenhoeck und Ruprecht, 1906), 43-45. Le travail de Wrede a donné lieu à une reprise critique ne remettant pas en cause la caractère secondaire d'He 13,22-25 de la part d'E. Burggaller, « Das literarische Problem des Hebräerbiefes », ZNW 9 (1908) : 110-131, et de Richard Perdelwitz, « Das literarische Problem des Hebräerbriefs », ZNW 11 (1910) : 59-78. [480]

${ }^{96}$ Erich Grässer, An die Hebräer. 1. Teilband (Hebr 1-6), EKK XVII/1 (Zürich : Benziger ; Neukirchen-Vluyn: Neukirchener, 1990), 17-18; An die Hebräer. 3. Teilband (Hebr 10,19-13,25), EKK XVII/3 (Zürich: Benziger; Neukirchen-Vluyn: Neukirchener, 1990), 409-410. Cesare Marcheselli-Casale, Lettera agli Ebrei. Nouva versione, introduzione e commento (Milano : Pauline, 2005), 642, considère aussi que la conclusion, qu'il qualifie de post-scriptum, ne peut être ni de Paul ni de l'auteur d'Hébreux.

97 Albert Vanhoye, Exegesis Epistulae ad Hebrces [ad usum privatum auditorum], Romæ: Pontificium institutum biblicum, 1968), 15. Traduction française proposée par Régis Burnet, «La finale de l'épître aux Hébreux : une addition alexandrine de la fin du II siècle ? », RB 120 (2013) : 423-440, ici 426. L'original latin est le suivant: $1^{\circ}$ Primum opus est prcedicatio longa, sedulo composita ut ore pronunciatur, et quae de facto pronuntiata in catibus christianis. Extenditur ab exordio usque ad primam conclusionem, scl. 13,21, excepto 13,19. $2^{\circ}$ Opus secundum est adscriptio brevis, stilo veloce, addito quando prodicatio ad alium coetum scripto missa est. Ho litterce constant una sententia ante perationem additis inserta et paucis aliis post perationem additis; in eis continentur nuntia et salutiones.

${ }_{98}^{9}$ Albert Vanhoye, La structure littéraire de l'épître aux Hébreux, préface du R. P. Stanislas Lyonnet S. J., Deuxième édition revue et augmentée (Paris : Desclée de Brouver, 1976), 220.

${ }^{99} \mathrm{He}$ 13,7.17.24.

${ }^{100}$ Vanhoye, La structure littéraire (note 98), 220.

${ }^{101}$ Vanhoye, La structure littéraire (note 98), 220.

${ }^{102}$ Vanhoye, La structure littéraire (note 98), 221.
} 
verbe qui signifie non pas tant écrire qu'envoyer un message ou mander, ce qui cadre

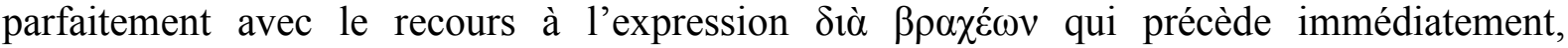
expression qui s'applique remarquablement au billet en lui-même sans qu'il soit nécessaire d'y voir quelque antiphrase pour désigner l'ensemble du discours. ${ }^{103}$ [481]

L'hypothèse telle qu'elle est formulée par Vanhoye a été reprise et précisée par Régis Burnet ${ }^{104}$ après que Gert Steyn ${ }^{105}$ eut introduit dans le débat le témoignage du Papyrus 46, auquel recourt aussi, nous l'avons vu, Clare Rotschild. ${ }^{106} \mathrm{Ce}$ papyrus, qui remonte, selon les spécialistes, entre 150 et 250 mais que l'on date le plus souvent, redisons-le, aux alentours de l'an 200, ${ }^{107}$ présente la particularité de contenir un recueil de lettres pauliniennes dans l'ordre suivant: Romains; Hébreux; 1 et 2 Corinthiens; Ephésiens; Galates; Philippiens ; Colossiens ; 1 Thessaloniciens. ${ }^{108}$ Hébreux y figure ainsi en deuxième position, ce qui peut surprendre mais trouve une explication dans le fait que les différentes écrits ont été classées par ordre de longueur décroissante et que, pour le copiste ou le modèle qu'il a suivi, qui n'aura pas voulu dissocier par ailleurs les deux épîtres aux Corinthiens, Hébreux devait appartenir pleinement au corpus paulinien. ${ }^{109}$ Pour Steyn, le fait qu'He13,22 commence en début de ligne après le $\dot{\alpha} \mu \eta ́ v$ qui conclut He 13,21 pourrait être le signe qu'He 13,22-25 constitue en fait une addition postérieure. Il reconnaît, dans un premier temps, que ce pourrait être là une simple coïncidence, ${ }^{110}$ mais fait de cette constatation, au fur et à mesure qu'il avance dans son raisonnement, un véritable argument en faveur du caractère secondaire d'He 13,22-25, alors que, selon nous, l'examen du papyrus [482] donne à penser qu'il n'y a, en aucun cas, nouveau paragraphe et que le copiste, arrivé en fin de ligne au terme de ce qui correspond désormais pour nous à He 13,21, a tout naturellement poursuivi au début de la ligne suivante sans qu'aucun espacement ne soit observable.

L'étude de Régis Burnet, qui reprend à son compte, outre les conclusions de Vanhoye, l'argument, inopérant selon nous, de Steyn, se sert également du $\mathrm{P}^{46}$ à un autre niveau tout en complétant l'argumentation de Vanhoye.

A la différence formelle que représente l'emploi de la première personne du singulier, Burnet ajoute le fait que les versets 24 et 25 « constituent une "seconde fin" alors que les versets 18 à

\footnotetext{
${ }^{103}$ Vanhoye, La structure littéraire (note 98), 221. [481]

${ }^{104}$ Burnet, « La finale de l'épître aux Hébreux » (note 97).

${ }^{105}$ Gert J. Steyn, « The Ending of Hebrews Reconsidered », ZNW 103 (2012) : 235-253.

${ }^{106}$ Voir supra, p. 474.

${ }^{107}$ Une fourchette comprise entre 200 et 225 est proposée sur le site de l'Institut für neutestamentliche Textforschung (http://ntvmr.uni-muenster.de/liste) de la Westfälische Wilhelms-Universität de Münster (consulté le 25 janvier 2017). La datation proposée par Philip W. Comfort et David P. Barrett, The Complete Text of the Earliest New Testament Manuscripts (Grand Rapids, MI : Baker Books, 1999), 193-197 (entre le début et le milieu du II siècle) paraît beaucoup trop haute.

${ }^{108}$ Pour une présentation de la discussion relative à l'ancienneté de ce papyrus, Steyn, «Ending » (note 105), 237. Le manuscrit lui-même, du moins ce qu'il contient de Rm, He, $2 \mathrm{Co}, \mathrm{Ep}, \mathrm{Ga}, \mathrm{Ph}$ et Col, est consultable, à partir de photographies d'une qualité remarquable, sur le site http://earlybible.com/manuscripts/p46.html (consulté le 27 janvier 2017). A l'origine, le papyrus 46, qui présente un certain nombre de feuillets manquants, devait contenir encore 2 Th (ainsi Bruce M. Metzger and Bart D. Ehrman, The Text of the New Testament. Its Transmission, Corruption, and Restauration [New York, Oxford: Oxford University Press, ${ }^{4} 2005$ ], 54) et peutêtre aussi Philémon (hypothèse envisagée par David C. Parker, An Introduction to New Testament Manuscripts and Their Texts [Cambridge : Cambridge University Press, 2008], 253). Jeremy Duff, « $\mathrm{P}^{46}$ and the Pastorals: A Misleading Consensus ? », NTS 44 (1998) : 578-590, a envisagé, quant à lui, que les Pastorales aient pu être incluses aussi, mais cela paraît peu vraisemblable en fonction de la taille initiale du papyrus qui comportait 54 feuillets (208 pages) dont neuf sont manquants (ces manques affectant de manière assez similaire le début et la fin du papyrus).

${ }^{109}$ Ainsi, notamment, Steyn, « Ending » (note 105), 238.

${ }^{110}$ Steyn, « Ending » (note 105), 238. [482]
} 
21 avaient déjà fait figure de conclusion, renforcée par un amen final $»{ }^{111}$ Pour ce qui est de la différence générique, le billet d'accompagnement, qu'il qualifie pour sa part de lettre, relève, de son point de vue, de la «lettre familière dont le but principal est l'échange de nouvelles et la manifestation à distance de sentiments ». ${ }^{112}$ Enfin, sur le plan du contenu, il constate que l'auteur, qui a jusque-là « conservé un anonymat volontaire », ${ }^{113}$ s'en départit, tout en prenant un recul certain pour rapport à l'écrit dans son ensemble en exhortant à supporter non pas «ma » ou « cette » lettre d'exhortation, mais «la » lettre d'exhortation ${ }^{114}$ (He 13,22). Il en infère, étendant à l'ensemble de la finale (He 13,22-25) une remarque que proposait Gräßer au sujet du verset 23 , que ladite finale est « une étiquette collée de manière bien visible sur Hébreux $\gg .{ }^{115}$

Il tire ensuite argument de l'histoire de la réception d'He pour préciser son hypothèse. Hormis les cinq versets qui y auraient été adjoints dans un deuxième temps, He aurait vu le jour à Rome. ${ }^{116}$ Nous ajouterions, pour notre part, que cela pourrait expliquer pourquoi, en milieu romain, on refusait la paternité paulinienne de l'écrit, dans la mesure où on aurait su depuis le départ que l'écrit n'était de Paul, ${ }^{117}$ tradition qui semble avoir été bien ancrée en milieu romain comme l'illustre le témoignage attribué par Jérôme à Gaïus dans son De viris inlustribus. ${ }^{118}$ Quant à l'intérêt nouveau manifesté pour l'écrit dans la sphère orientale à partir de la fin du II siècle, intérêt qui se conjugue avec l'attribution à Paul de la paternité de l'écrit, il s'expliquerait par l'intervention de la «seconde main » qui, " en adjoignant une sorte de condensé de paulinisme au sermon, [a] [483] fait brusquement passer l'écrit dans l'orbe paulinien ». ${ }^{119}$ Il ajoute ceci : "Manifestement consciente que ce texte est connu à Rome, la "seconde main" prend bien soin de faire saluer "ceux d'Italie" qui paraissent entourer "Paul" : cela lui permet d'expliquer pourquoi on ne l'a pas connu plus tôt et pourquoi les Romains en ont déjà entendu parler $\gg{ }^{120}$ Le $\mathrm{P}^{46}$, dont on sait que l'origine est à chercher en Egypte, serait un témoin précieux de ce tournant, dès lors qu'il comporte le billet additionnel et qu'il range He parmi les lettres pauliniennes. Sa datation autour de l'an 200 permettrait de situer l'ajout du billet dans la sphère alexandrine à peu près à ce moment-là.

Cette hypothèse pourra peut-être paraître trop précise. Il n'en demeure pas moins qu'elle permet de rendre compte à la fois de la réception très précoce d'He à Rome, de la nonreconnaissance de la paternité paulinienne dans la capitale de l'Empire, et du retournement qui s'opère en Orient vers la fin du II siècle. Par ailleurs, elle pourrait contribuer à expliquer, en fonction de l'ordonnancement particulier des épîtres au sein du $\mathrm{P}^{46}$, les conditions et les circonstances dans lesquelles ce qui a toutes les apparences d'un billet additionnel visant à rattacher $\mathrm{He}$ à la tradition paulinienne a été accolé au premier écrit, en lequel ce billet reconnaît à juste titre « un discours d'exhortation » qui n'a rien, quant à lui, d'épistolaire. Le $\mathrm{P}^{46}$ doit donc être tenu pour un témoin spécifique - et - en l'état de nos connaissances et des découvertes papyrologiques qui ont été faites - unique de l'intérêt qui a été manifesté pour He en tant qu'écrit "paulinien » à la fin du $\mathrm{II}^{\mathrm{e}}$ siècle. Cela étant, il est tout à fait possible que l'adjonction du billet remonte plus haut dans le temps que le $\mathrm{P}^{46}$ lui-même.

\footnotetext{
${ }^{111}$ Burnet, « La finale de l'épître aux Hébreux » (note 97), 424.

${ }^{112}$ Burnet, « La finale de l'épître aux Hébreux » (note 97), 424-425.

${ }^{113}$ Burnet, « La finale de l'épître aux Hébreux » (note 97), 426.

${ }^{114}$ Grässer, An die Hebräer. 3. Teilband (note 96), 412.

${ }^{115}$ Burnet, «La finale de l'épître aux Hébreux » (note 97), 429.

${ }^{116}$ Burnet, « La finale de l'épître aux Hébreux » (note 97), 429-432.

${ }^{117}$ Ainsi déjà Spicq. Voir supra, note 36.

${ }^{118}$ Voir supra, note 37. [483]

${ }^{119}$ Burnet, « La finale de l'épître aux Hébreux » (note 97), 434.

${ }^{120}$ Burnet, « La finale de l'épître aux Hébreux » (note 97), 434-435.
} 
Pour conclure, les arguments très forts apportés déjà et plus particulièrement par Vanhoye à l'appui du caractère additionnel et secondaire de ce billet nous paraissent être les plus forts et inviter à considérer que c'est dans un deuxième temps, et sans doute en milieu égyptien ${ }^{121}$ et non pas à Rome même, ${ }^{122}$ qu'He a été rattaché à la nébuleuse paulinienne pour faciliter son intégration au sein de ce qui allait devenir le canon scripturaire. ${ }^{123}$

${ }^{121}$ La contribution de Burnet est, à cet égard, tout à fait importante.

${ }^{122}$ L'hypothèse de Backhaus garde toute sa pertinence pour expliciter en quoi le discours lui-même pourrait trouver un ancrage dans une communauté de maison romaine, mais échoue, nous semble-t-il, à rendre compte pleinement de la singularité du billet additionnel.

${ }^{123}$ On retrouve ainsi l'intuition fondamentale de Grässer.

Nous tenons à exprimer notre reconnaissance envers Jean-Daniel Kaestli, qui, au cours du colloque, nous a rendu plus particulièrement sensible à ce qui pouvait inviter à voir en He 13,22-25 un billet additionnel. Ses remarques, comme celles de Jörg Frey et de Pierre de Salis, nous ont incité à reprendre en profondeur le texte de notre intervention. 